\title{
Considerações sobre a tendência da tuberculose no Brasil
}

\author{
Dina Czeresnia Costa $\quad$ *Campanha Nacional Contra \\ a Tuberculose - MS.
}

Este trabalho analisa os vários aspectos que influenciam no padrão da mortalidade e morbidade da tuberculose: a variação nas condições de vida, os efeitos da quimioterapia e a dinâmica de transmissão da doença.

Caracteriza os indicadores utilizados na medida da tuberculose que são construidos a partir de inquéritos de prevalência e dos dados de notificação. Os principais indicadores são: risco de infecção; mortalidade; coeficiente de incidência de meningite tuberculosa; coeficientes de incidência de casos de tuberculose, especialmente os pulmonares baciliferos, etc.

$O$ trabalho analisa dados recentes de incidência de casos pulmonares baciliferos nos estados do Pará e Rio Grande do Sul e mortalidade por tuberculose nas capitais do Pará, Rio Grande do Sul, Pernambuco e Rio de Janeiro, que revelam uma tendência à queda, ainda que em ritmo diferenciado. Discute-se a maneira que a crise econômica atual do pais repercutiria no número de casos e mortes pela doença.

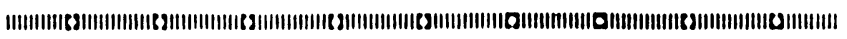

Este trabalho tem como objetivo estudar alguns aspectos da epidemiologia da tuberculose. Discute a relação entre crise econômica e incidência da tuberculose, a partir da análise de dados recentes sobre a doença. Analisa também as limitações dos indicadores utilizados na medida da tuberculose especificamente no Brasil.

Os dados disponíveis são ainda incipientes, porém permitem algumas reflexões sobre a tendência da tuberculose $\mathrm{e}$ sobre a utilização dos indicadores da doença em nosso país.

\section{PADRÃO DE VIDA E TUBERCULOSE}

A tuberculose representa sério problema de saúde no Brasil, pelo grande número de pessoas que atinge. Sendo uma doença fortemente favorecida pela precariedade das
Recebido para publicação em $20 / 05 / 85$. 
condiçōes de vida, uma questão permanentemente discutida é a sua tendência em conjunturas de aguda crise econômica e social, como a que vivencia o país atualmente. Esta é uma questão difícil de ser respondida. É preciso chamar a atenção sobre os vários aspectos que influenciam no padrão de morbidade e mortalidade da tuberculose, em diferentes épocas e sociedades. Seria incorreto estabelecer uma relação de determinação mecânica entre crise econômica, deterioração da qualidade de vida e aumento dos casos e mortes por tuberculose, sem uma análise minuciosa desses aspectos.

É de conhecimento quase de senso comum que a tuberculose é extremamente vulnerável às variações no padrão de vida. Por exemplo, diferentes autores registraram o crescimento da doença durante o primeiro período da revolução industrial (fins do século XVIII e início do século XIX), na Europa, em função da acentuada deterioração das condições de vida da classe trabalhadora. A tuberculose cresceu no início da revolução industrial, quando o desenvolvimento da produção fabril nas cidades obrigava os trabalhadores a morarem aglomerados e as condições de trabalho eram insalubres e desgastantes ${ }^{3}$. No entanto, registrou-se expressiva queda da mortalidade por tuberculose, a partir de fins do século XIX. Mckeown analisou essa mudança, na Inglaterra, e apontou as possíveis razões para a diminuição do número de mortes. Para ele, antes da introdução da quimioterapia, após a 2 a guerra mundial, as medidas médicas pouco influíram para a queda na mortalidade. Em relação à hipótese de variação na virulência do bacilo, em relação à população inglesa, Mckeown afirma ser impossivel ter ocorrido uma variação que exercesse uma influência tão significativa na mortalidade. Já em relação as variações do meio social, ocorreriam, durante a consolidação da revolução industrial, determinadas mudanças no padrão de vida, principalmente nas condições de alimentação, que são apontadas como a causa principal da diminuição da mortalidade na Inglaterra ${ }^{11}$.

Essas observações de Mckeown encontram fundamento em Frost, quando este afirmou que "nada teve mais influência sobre o declínio da tuberculose que a progressiva melhoria na ordem social" e que "um dos aspectos mais essenciais no efetivo controle da doença é a melhoria do padrão de vida dos estratos econômicos mais baixos"'6.

No Brasil, a tuberculose apresentou sempre grande mortalidade, e o contexto que favorece seu desenvolvimento e distribuição foi percebido nos primeiros relatos da enfermidade. Lourival Ribeiro revela que, na época colonial, médicos e legistas apontaram os gravíssimos problemas decorrentes da disseminação da doença entre as "classes desvalidas", 
especialmente entre os escravos ${ }^{16}$. Estudos contemporâneos, como os de Ruffino e P. Pereira ${ }^{18}$ e Guimarães ${ }^{6}$, analisam a queda da mortalidade por tuberculose no Rio de Janeiro, a partir de 1860 , e também ressaltam o peso das mudanças sociais nesse processo.

A partir da introdução dos quimioterápicos, contudo, os estudos têm destacado a importância das medidas médicosanitárias para o decréscimo da mortalidade. $O$ uso da quimioterapia coincidiu, efetivamente, com o aumento no ritmo da queda da mortalidade pela tuberculose no Brasil e em vários países desenvolvidos.

Já estudo realizado na Holanda, centrado na noção de risco de infecção, demonstrou que o aumento da tendência de queda da tuberculose ocorreu mesmo antes da introdução da quimioterapia, o que foi atribuído também a uma medida específica: a pasteurização compulsória do leite a partir de 1940, que eliminou a tuberculose de transmissão bovina ${ }^{5}$.

Outro fator que também influi na tendência da tuberculose é a própria dinâmica de transmissão da doença. Quando existe pequeno número de fontes de contágio - em uma situação em que a população desfruta de um bom padrão de vida - ocorre uma forte tendência de redução da doença. Quando existe grande número de fontes de contágio, o risco de infecção é al to, e o ritmo de redução do problema é muito lento ${ }^{8}$.

Estes fatos sublinham a dificuldade em atribuirmos a uma ou a outra causa, isoladamente, o desenvolvimento e distribuição da tuberculose nas sociedades contemporâneas. Outro aspecto relevante a ser considerado refere-se aos próprios indicadores utilizados para medir a tuberculose. Cabe discutir as limitações e dificuldades que apresentam, quando usados para avaliar a magnitude da tuberculose, principalmente no Brasil.

\section{A UTILIZAÇÃO DOS INDICADORES DA TUBERCULOSE}

Os indicadores podem ser construídos através dos dados de notificação ou de inquéritos de prevalência. Os dados de notificação dependem fundamentalmente da qualidade do sistema de informação do país. Já os inquéritos de prevalência se constituem através de exames radiológicos, baciloscopia de escarro, testes tuberculínicos realizados em amostras representativas da população. Além de informarem sobre a prevalência da doença, tornam possível a estimativa do risco de infecção e oferecem várias outras informações operacionais. Porém, somente quando realizados repetidamente, possibilitam a avaliação de tendência. Esses inquéritos são extremamente trabalhosos e caros. Os estudos longitudinais 
(coorte) permitem uma avaliação ainda melhor da magnitude do problema, porém são ainda mais custosos e tecnicamente difíceis. Ainda assim, foram realizados em algumas regiões da Índia ${ }^{19}$.

$\mathrm{Na}$ década de 50 , foram desenvolvidos inquéritos de prevalência em vários países, financiados pela OMS, particularmente em pequenos países africanos. Em países asiáticos (Coréia, Japão, Filipinas, Malásia, Singapura, etc) foram também realizados repetidos inquéritos, apesar das dificuldades técnicas e do al to custo ${ }^{5}$.

No Brasil, devido a dificuldades técnicas, altos custos associados ao tamanho da população, os inquéritos de prevalência são de difícil realização. Porém, inquéritos de prevalência em grupos populacionais específicos podem dar informações úteis para o planejamento e avaliação de programas.

Os principais indicadores utilizados para medir a tuberculose são:

a) RISCO DE INFECÇÄO TUBERCULOSA - indica a proporção da população que foi infectada com o bacilo da tuberculose no curso de um ano e é geralmente expresso como percentagem ou como taxa. A medida do risco de infecção, em anos sucessivos, permite uma avaliação da mag. nitude e tendência da tuberculose.

K. Styblo, S. Meijer e Ian Sutherland desenvolveram um modelo matemático que, utilizando informações de vários inquéritos tuberculínicos, na Holanda, construíram séries de riscos anuais de infecção tuberculosa. Os autores criaram um método que possibilitasse a aplicação deste modelo aos resultados de inquéritos tuberculínicos, em amostras representativas da população, em uma determinada idade, nos demais países 20 .

O risco de infecção, por utilizar apenas o teste tuberculínico na sua avaliação e por não depender da qualidade dos serviços de controle, é considerado um indicador confiável. Contudo, não pode ser estimado em países onde o BCG intradérmico é administrado amplamente, pois não há como distinguir a alergia provocada pelo bacilo da tuberculose da provocada pela vacina.

Styblo, analisando comparativamente os dados de risco de infecção, incidência e prevalência de bacilíferos e mortalidade, em países onde foram realizados inquéritos de prevalência, encontrou uma relação relativamente constante entre esses indicadores. Sustentou que, nos países em desenvolvimento, $1 \%$ do risco de infecção corresponde a 55 casos de tuberculose pulmonar baciliferos por 100.000 habitantes ao ano 21 .

Estas relações são úteis na estimativa dos casos que seriam epidemiologicamente esperados, fornecendo parâme- 
tros para avaliação operacional de programas de controle da tuberculose. No entanto, devem ser utilizadas com cautela, pois constituem generalizações passíveis de erros importantes, como super ou subestimativa do problema.

No Brasil, foi realizada uma investigação da prevalência da infecção tuberculose, de 1970 a 1973, em uma amostra de escolares de 6 a 9 anos que ingressaram no primeiro ano das escolas das capitais.

Este trabalho trouxe informações para a avaliação da situação epidemiológica da tuberculose no país, ao demonstrar a diferença na magnitude da tuberculose entre as regiões, que aumenta do Sul para o Norte ${ }^{1}$. (Ver quadro I).

\section{QUADRO I}

Prevalência de infecção tuberculosa em escolares de 9 capitais brasileiras (Reatores fortes ao teste tuberculínico - PPD).

1971

\begin{tabular}{lc}
\hline Capitais & $\begin{array}{c}\text { \% de Reatores fortes } \\
\text { ao PPD (10 mm ou +) }\end{array}$ \\
\hline Manaus & 19,6 \\
Belém & 25,9 \\
Fortaleza & 16,1 \\
Recife & 13,6 \\
Salvador & 20,5 \\
São Paulo & 6,6 \\
Curitiba & 6,4 \\
Cuiabá & 9,7 \\
Brasília & 8,5 \\
\hline
\end{tabular}

In: Almeida P...Aristides: Prevalência da infecção tuberculosa em escolares das capitais brasileiras Revista da DNT, vol. 17, no 66, abril/jun. 1973.

Este inquérito de prevalência de infecção apresentou algumas limitações. Em relação à composição da amostra estudada, que não pode ser considerada como representativa da população nos diversos Estados; e ao curto período estudado, que dificulta um cálculo da tendência do risco de infecção. Os dados fornecidos possibilitaram, porém, uma estimativa utilizável para uma avaliação operacional do programa de controle da tuberculose.

O risco de infecção estimado para o Brasil foi de 1,5\%, em 1973 (com variaçōes entre as regiōes). Com base nas observações de Styblo já descritas, estimou-se a incidência anual em 120.000 casos de todas as formas de tuberculose. Assumiu-se, a partir de então, uma redução anual do risco de $8 \%$, entre 1973 e 1976, e 5\%, a partir de 197714,15 . 
Com o decorrer do tempo, esses parâmetros vão se tornando cada vez mais difíceis de serem utilizados, pois não podem ser reavaliados através de outros inquéritos de prevalência de infecção, devido à ampla cobertura de vacinação de BCG intradérmico em crianças menores de 1 ano. Somente no Rio Grande do Sul, que só vacina crianças em idade escolar, realizou-se recentemente um estudo de prevalência de infecção tuberculosa. Encontrou-se uma prevalência de $3,01 \%$ em crianças de 7 anos e estimou-se o risco de infecção em $0,4 \%^{17}$.

b) MORTALIDADE - a taxa de mortalidade, como já foi visto, foi um indicador extremamente útil no estudo da tuberculose nas sociedades industriais. A partir da introdução da quimioterapia, que influi decisivamente na mortalidade, este indicador tendeu a relacionar-se mais com a eficiência dos serviços do que propriamente com a situação epidemiológica da tuberculose ${ }^{9}$. A sua comparação com os outros indicadores é ainda importante na análise epidemiológica.

c) COEFICIENTE DE INCIDENCIA DE MENINGITE TUBERCULOSA EM MENORES DE 5 ANOS - é um indicador importante no estudo da epidemiologia da tuberculose, porque, na faixa etária de 0 a 4 anos, está diretamente relacionado com a incidência de outras formas de tuberculose. Os casos são graves e, presumivelmente, tendem a ser encaminhados aos serviços de saúde. Sustenta-se também que estão diretamente relacionados à baixa cobertura de BCG na faixa etária de 0 a 1 ano ${ }^{12}$.

Os problemas que dificultam o uso do coeficiente de incidência da meningite tuberculosa para avaliar a magnitude e tendência da tuberculose são: as grandes dificuldades de diagnóstico; a baixa freqüência e irregular notificação; e, em função do pequeno número de casos, qualquer variação do número absoluto reflete-se acentuadamente no coeficiente. Ainda assim, é um indicador útil para avaliação operacional.

d) COEFICIENTE DE INCIDENCIA DE CASOS DE TUBERCULOSE - é considerado teoricamente um bom indicador da magnitude e tendência da tuberculose, embora também apresente algumas limitações. Como a tuberculose é uma doença de longa duração, o caso, quando diagnosticado e notificado, pode já se ter iniciado há algum tempo. Assim, a incidência se confunde com a prevalência. Apesar disso, havendo uma proporção alta e constante de "casos novos", esta incidência pode ser tomada como um indicador consistente da tendência da tuberculose. É também 
necessária a existência de um programa abrangente de controle de tuberculose e de um sistema de informação, com critérios padronizados de registro e notificação, para que esses dados sejam confiáveis.

Entre os casos notificados, os confirmados bacteriologicamente têm uma importância especial porque, apesar de não oferecerem uma dimensão total do número de pessoas doentes, são os considerados capazes de contagiar outros indivíduos e, assim, reproduzir a doença.

Bulla, ao estudar comparativamente os casos de tuberculose notificados em diversos países, afirma que, devido à diferença de critérios utilizados no mundo para notificar os casos e à pequena importância estatística dos casos confirmados bacteriologicamente, o coeficiente de incidência é também um indicador de difícil utilização ${ }^{4}$.

\section{TENDENCIA DA TUBERCULOSE NO BRASIL}

Em nosso país, houve uma expansão significativa das atividades de controle da tuberculose durante a década de 70 . $\mathrm{O}$ número de municípios com atividades de controle da doença ampliou-se de 405 para 2754 em 1981, considerando-se como população coberta toda aquela residente em municípios com pelo menos um serviço de controle, sem discutir criticamente a acessibilidade ou poder resolutivo do serviço ${ }^{14,15}$.

$\mathrm{O}$ número de casos diagnosticados no país também cresceu durante esse período, traduzindo não um aumento do problema, mas o resultado da expansão do programa de controle (quadro II).

\section{QUADRO II}

Número de casos de tuberculose notificados no Brasil 1973 - 1983

\begin{tabular}{lccccccccccc}
\hline $\begin{array}{c}\text { Ano } \\
\text { n } \text { Casos }\end{array}$ & 1973 & 1974 & 1975 & 1976 & 1977 & 1978 & 1979 & 1980 & 1981 & 1982 & 1983 \\
\hline Brasil & 45665 & 46987 & 53419 & 51301 & 54552 & 57821 & 65064 & 70596 & 86411 & 87824 & 86617 \\
\hline
\end{tabular}

In: Programa Nacional de Controle de Tuberculose: Relatório de Avaliação das atividades realizadas em 1983: DNPS, CNCT, INAMPS, Mimeo.

Concomitante a essa expansão, foi criado um sistema de informações para onde são enviados os casos notificados em vários níveis dos serviços de saúde (secretarias municipais, estaduais e INAMPS). Este sistema foi implantado progressivamente: em 72, no Espírito Santo; em 75, no Rio Grande do Sul, Ceará, Distrito Federal, Acre e Rondônia; em 76, no Pará; em 79, no Piauí e, finalmente, em 1981, cobre todo o país ${ }^{14,15}$. 
Desse modo, a análise da incidência dos casos deve obrigatoriamente ser relacionada com as características operacionais dos serviços envolvidos no controle da tuberculose. Nos Estados onde a expansão dos serviços e a criação de um sistema único de informação ocorrem há mais tempo, é possível a análise de uma série temporal mais longa. Para o país como um todo, somente a partir de 1981 é possivel fazer uma análise, o que é um período muito curto para permitir inferências minimamente consistentes.

Outro aspecto a ser ressaltado é a qualidade operacional do serviço e a do sistema de informação. Os Programas de Controle da Tuberculose nos Estados do Rio Grande do Sul e do Pará são considerados, segundo os relatórios de avaliação da Divisão de Pneumologia Sanitária, como os de melhor qualidade nesses dois aspectos, possibilitando os melhores dados para análise da incidência de casos no país atualmente.

GRÁFICO I

COEFICIENTE DE INCIDENCIA DE CASOS DE TUBERCULOSE PULMONAR BACILIFERA/ 100000

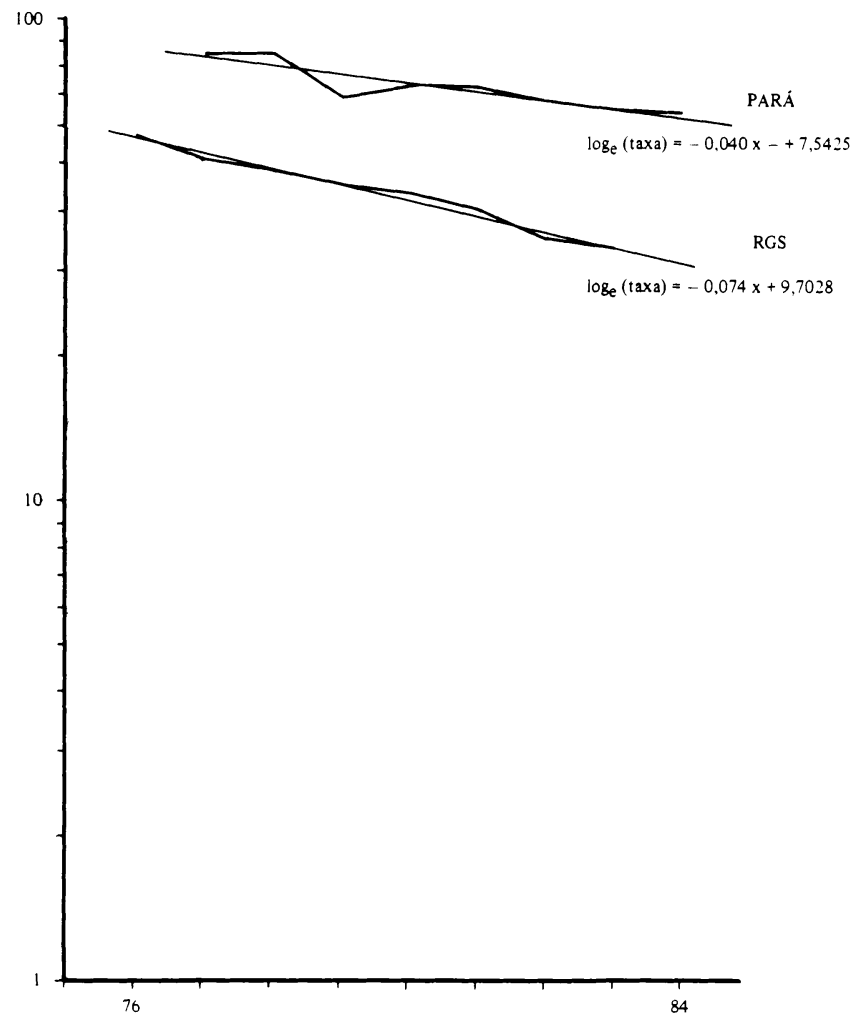


O gráfico I mostra a incidência notificada de tuberculose pulmonar bacilífera no Pará e Rio Grande do Sul, nos últimos 8 a 9 anos. Esses coeficientes foram construídos a partir dos dados de notificação oficiais da Divisão de Pneumologia Sanitária, de 1978 em diante. Os dados de 76 e 77 foram formecidos pelas respectivas Secretarias de Saúde. A população utilizada como denominador foi a fornecida pela Divisão de Epidemiologia do Ministério da Saúde, por estar corrigida em relação aos censos de 70 e 80 , o que é fundamental para a análise de tendência na série temporal em questão. O gráfico foi construído em escala semilogarítmica.

No Pará, esses coeficientes variam de 85.5/100000, em 77 , a 64.8/100000, em 84. Estes dados confirmam a extensa magnitude da doença nesse Estado (estima-se que os casos bacilíferos representem cerca de $60 \%$ do total de casos). Já no Rio Grande do Sul, apesar de ainda apresentar uma taxa alta, a magnitude do problema é bem menor (57.9/ 100000 , em 76, e 31.3/100000, em 84). Esta diferença nos coeficientes dos dois Estados é coerente com o comportamento de outros indicadores de saúde, como mortalidade infantil e esperança de vida ao nascer, refletindo variações nas condições sociais e econômicas entre as regiões Norte e Sul do país, fato observado em diferentes estudos $7,10,13$.

GRÁFICO II

RISCO ANUAL ESTIMADO DE INFECÇÃO TUBERCULOSA $1950-1971$ e 195

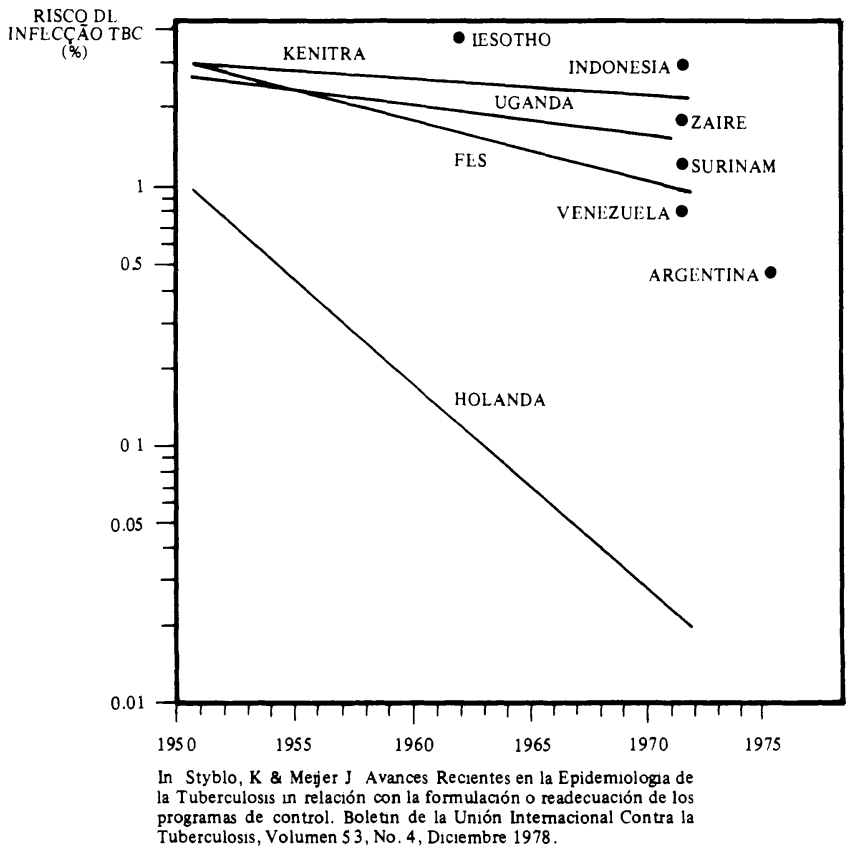

Cadernos de Saúde Pública, R.J., 1 (3) : 313-326, jul/set, 1985. 
A tendência de queda observada, obtida através de regressão linear sobre os logaritmos das taxas anuais, também é maior no Rio Grande do Sul (7,4\% ao ano) do que no Pará (4,0\% ao ano), refletindo da mesma forma diferenças no padrão de vida das populações dos dois Estados. Como vimos, a tendência de queda da tuberculose é observada histórica ou mesmo secularmente em diversos países do mundo. O ritmo desta queda é que varia muito, de acordo com o grau de desenvolvimento e padrão de vida. No gráfico II, observamos a variação da tendência da tuberculose, através do risco de infecção, em diferentes países subdesenvolvidos, comparados com a da Holanda.

As taxas de mortalidade por tuberculose, apesar da deficiência dos registros característicos de nosso país, também demonstram uma tendência de queda. Os gráficos III e IV mostram a evolução da mortalidade nas cidades de Belém, Porto Alegre, Recife e Rio de Janeiro. A tendência da queda da mortalidade em Belém (12,4\% ao ano) e Porto Alegre ( $12,4 \%$ ao ano) é bem maior que a da incidência nos Estados correspondentes.

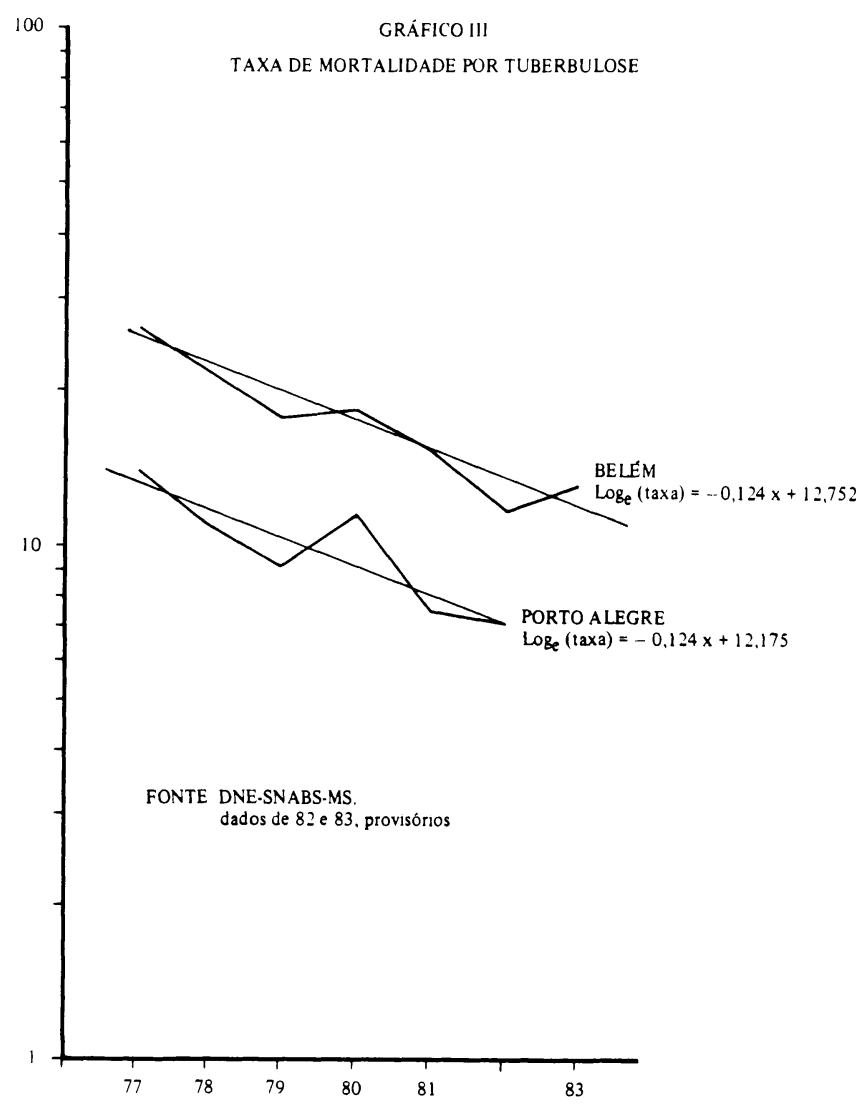


(iRAHICOIV

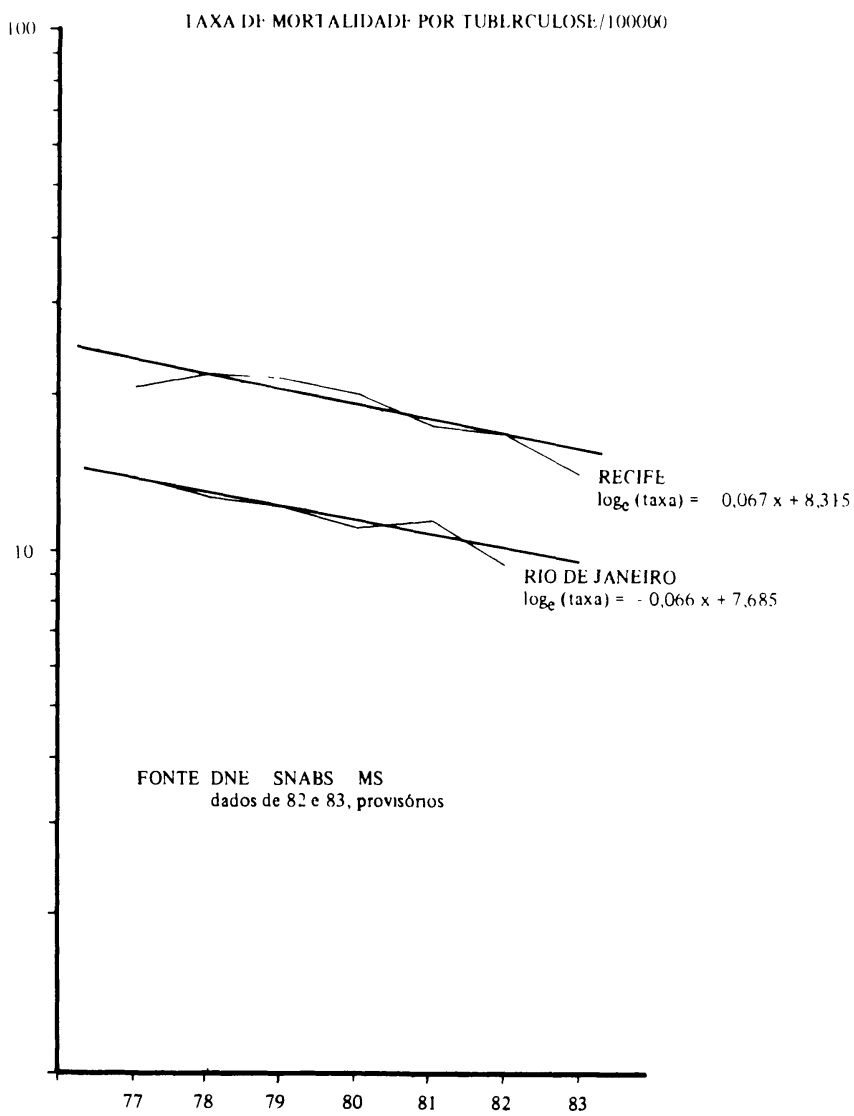

A mortalidade proporcional por tuberculose, apesar de ser um indicador dependente da estrutura etária e muito sensivel a modificações nos outros grupos de causas de óbito, também indica uma queda. Observamos, da mesma forma que para a incidência, maior dimensão na mortalidade em cidades do Norte do que nas do Sul.

\section{QUADRO II}

Mortalidade proporcional por tuberculose em algumas capitais brasileiras

\begin{tabular}{|c|c|c|c|c|c|c|c|}
\hline Capital & 1977 & 1978 & 1979 & 1980 & 1981 & 1982 & 1983 \\
\hline $\begin{array}{l}\text { Belém } \\
\text { Recife } \\
\text { Rio de Janeiro } \\
\text { Porto Alegre }\end{array}$ & $\begin{array}{l}3,4 \\
2,5 \\
1,7 \\
1,9\end{array}$ & $\begin{array}{l}2,7 \\
2,6 \\
1,6 \\
1,6\end{array}$ & $\begin{array}{l}2,2 \\
2,6 \\
1,5 \\
1,3\end{array}$ & $\begin{array}{l}2,4 \\
2,4 \\
1,3 \\
1,7\end{array}$ & $\begin{array}{l}2,2 \\
2,3 \\
1,4 \\
1,1\end{array}$ & $\begin{array}{l}1,8 \\
2,2 \\
1,3 \\
-\end{array}$ & $\begin{array}{l}2,1 \\
1,9 \\
- \\
-\end{array}$ \\
\hline
\end{tabular}

Fonte: DNE - SNABS - MS.

Cadernos de Saúde Pública, R.J., 1 (3): 313-326, jul/set, 1985. 
Ao compararmos a queda da mortalidade em Belém e Porto Alegre com a do Recife (6,7\% ao ano) e Rio de Janeiro $(6,6 \%$ ao ano), reafirma-se a importância da qualidade dos serviços neste aspecto. De fato, o controle da tuberculose nas duas primeiras cidades é considerado melhor do que nas duas últimas, e a tendência da mortalidade confirma esta tese.

\section{CONCLUSÕES}

Os dados de notificação disponíveis sobre a tuberculose nos anos recentes indicam uma tendência de queda do problema, apesar da doença manter-se como sério problema de saúde pública. Esses dados não permitem afirmar a hipótese de haver uma relação direta entre a crise econômica atual e aumento do número de casos e mortes pela doença.

Ainda assim, vários trabalhos demonstram, sem dúvida, uma relação direta entre indicadores de saúde e indicadores sociais como renda, deterioração do salário real, PIB, saneamento, educação, etc. Os indicadores de saúde mais relacionados nesses estudos são mortalidade infantil, esperança de vida ao nascer, mortalidade por doenças infecciosas, doenças degenerativas (hipertensão arterial, algumas formas de câncer, doenças profissionais) e acidentes de trabalho ${ }^{2}$.

Contudo, a tuberculose, apesar de ter um comportamento muito vulnerável às variações no padrão de vida, como já foi visto nos estudos sobre o passado, apresenta uma dinâmica de transmissão lenta. Provavelmente bem mais lenta atualmente do que no passado, devido a transformações sociais, tanto no nível do conhecimento e intervenção médica, como nas mudanças históricas do padrão de vida das populações nas sociedades de economia industrial. Essa dinâmica de transmissão lenta faz com que as repercussões da crise econômica e social sobre a doença possivelmente apareçam refletidas mais tardiamente. Estas repercussões devem ser estudadas não somente a partir do aumento dos coeficientes, mas também a partir da diminuição da velocidade de queda do problema.

De fato, o estudo da dinâmica epidemiológica de cada doença é fundamental para que não se estabeleçam relações mecânicas entre os indicadores de saúde específicos e os indicadores sociais obtido em determinadas conjunturas.

Os dados disponíveis até o momento são, infelizmente, ainda muito restritos, não permitindo maiores inferências em relação a essa discussão. Certamente, poderemos estudar melhor estes aspectos no decorrer dos próximos anos, quando dispusermos de dados mais abrangentes. Algumas outras hipóteses podem ser, contudo, reforçadas: 
- o ritmo de queda da incidência da tuberculose, mais lento ou mais rápido, pode ser relacionado com diferenças no desenvolvimento e padrão de vida das populações.

- já o ritmo de queda da mortalidade por tuberculose parece estar mais fortemente relacionado com a eficiência dos serviços de controle da doença.

Tendo em vista as limitações dos indicadores, o estudo da incidência da tuberculose pulmonar bacilífera e da mortalidade por tuberculose - quando associado a uma cuidadosa análise da operação dos serviços de saúde e do sistema de informação - é o indicador mais adequado na análise epidemiológica e operacional da doença em nosso país, atualmente.

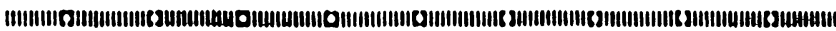

This paper analises the various factors which affect the rate of mortality and illness from tuberculosis i.e. the variation in the quality of life of the people affected, the effects of chemotherapy and the dynamics of the transmission of the disease.

It describes the epidemiological indices used to measure the spread of tuberculosis. These indices are built up from inquiries of prevalence of the disease and the number of notified cases. The main indices are: the risk of infection, mortality, the coefficient of the incidence of tubercular meningitis, the coefficient of the incidence of cases of tuberculosis, especially smear positives, etc.

The paper also analises recent data of incidence of cases of smear positives in the states of Para and Rio Grande do Sul and the mortality rate from tuberculosis in the state capitals of Pará, Rio Grande do Sul, Pernambuco and Rio de Janeiro, which reveal a downward trend, thought at different rates. The nature of how the present economic crisis in the country is affecting the number of cases and deaths as a result of the disease is also discussed.

\section{REFERÊNCIAS BIBLIOGRÁFICAS}

1. ALMEIDA, A. P. de. Prevalência da infecção tuberculosa em escolares das capitais brasileiras: relatório progressivo. $R$. Div. nac. Tuberc., 17 (66): 155-74, abr./jun. 1973.

2. AROUCA, A.T. A análise dos determinantes das condições de saúde da população brasileira. In: GUIMARÃES, R. org. Saúde e trabalho no Brasil: contribuição para um debate. Rio de Janeiro, Graal, 1975. 
3. BERlingueR, G. Medicina e politica. São Paulo, CEBES/ HUCITEC, 1978.

4. BULLA, A. Cuantos enfermos de tuberculosis hay en el mundo? Cron. OMS, 31: 311-9, 1977.

5. DAM, H.G. \& PIO, A. Epidemiological research in tuberculosis control. Genève, WHO, 1983.

6. FROST, W.H. How much control of tuberculosis. In: PAPERS of Wade Hampton Frost. New York, Commonwealth Fund, 1941, p. 611.

7. FUNDAÇÃO INSTITUTO BRASILEIRO DE GEOGRAFIA E ESTATISTICA. Indicadores sociais: relatório 1979. Rio de Janeiro, IBGE, 1979. 441 p.

8. GERHARDT, G. Tuberculose. Tema, Projeto Radis, 3: 1-7, 1982.

9. GUIMARÃES, R. Determinação social e doenças endêmicas: o caso da tuberculose. Rio de Janeiro, 1982. mimeo.

10. INSTITUTO BRASILEIRO DE ANÁLISES SOCIAIS. Saúde $e$ trabalho no Brasil. Petrópolis, Vozes, 1982. 128 p.

11. MCKEOWN, T. \& LOWE, C.R. Introducción a la medicina social. México, Siglo Veintiuno, 1981. cap. 1.

12. PIO, A. The effectiveness of BCG vaccination against tuberculosis Napoli Curse. 1982. mimeo.

13. POSSAS, C. Saúde e trabalho: a crise da previdência social. Rio de Janeiro, Graal, 1981. 324 p.

14. PROGRAMA NACIONAL DE CONTROLE DA TUBERCULOSE. Relatório das atividades realizadas em 1981. DNPS, INAMPS, mimeo.

15. PROGRAMA NACIONAL DE CONTROLE DA TUBERCULOSE. Relatório de avaliação das atividades realizadas em 1983. DNPS, CECT, INAMPS.

16. RIBEIRO, L. A luta contra a tuberculose no Brasil. Rio de Janeiro, Sul Americana, 1956.

17. RIO GRANDE DO SUL. Secretaria de Saúde e do Meio Ambiente. Estudo da prevalência de infecção tuberculosa na população escolar de 6.7 anos no Rio Grande do Sul. Porto Alegre, 1984. mimeo.

18. RUFFINO, N.A. \& PEREIRA, J.C. Mortalidade por tuberculose e condiçôes de vida: o caso Rio de Janeiro. Debate (12): 27 $34,1981$.

19. SHIMAO, T. Las encuestas de prevalencia de la tuberculosis. Bol. Union Inter. Contra Tuberc., 57 (2):127-33, jun. 1982.

20. STYBLO, K.; MEIJER, J. \& SUTHERLAND, I. The transmission of tubercle bacilli, its trend in human population. Bull. Union Inter. Against Tuberc., 42: 5-104, 1969.

21. STYBLO, K. \& ROUILON, A. Estimaciones de la incidencia mundial de la tuberculosis pulmonar in baciloscopia positiva: no fiabilidad de las cifras informadas oficialmente. Bol. Union Inter. Contra Tuberc., 56 (3/4): 128-37, 1981.

Agradeço a colaboração de Paulo C. R. Barata e de Nilson do Rosário Costa na realização deste trabalho. 